\title{
A contextual extension of Spekkens' toy model
}

Jan-Åke Larsson

The self-archived postprint version of this journal article is available at Linköping University Institutional Repository (DiVA):

http:/ / urn.kb.se/ resolve?urn=urn:nbn:se:liu:diva-87981

N.B.: When citing this work, cite the original publication.

Larsson, J., (2012), A contextual extension of Spekkens toy model, AIP Conference Proceedings, Vol. 1424, pp. 211-220. https:// doi.org/ 10.1063/ 1.3688973

Original publication available at:

https:// doi.org/ 10.1063/1.3688973

Copyright: AIP Publishing

http:// www.aip.org/ 


\section{A contextual extension of Spekkens' toy model}

Cite as: AIP Conference Proceedings 1424, 211 (2012); https://doi.org/10.1063/1.3688973

Published Online: 29 March 2012

Jan-Åke Larsson

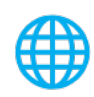

\section{Conference Proceedings}

\section{Get $30 \%$ off all print proceedings!}




\title{
A contextual extension of Spekkens' toy model
}

\author{
Jan-Åke Larsson \\ Institutionen för Systemteknik, Linköpings Universitet, SE-581 83 Linköping, Sweden
}

\begin{abstract}
Quantum systems show contextuality. More precisely, it is impossible to reproduce the quantum-mechanical predictions using a non-contextual realist model, i.e., a model where the outcome of one measurement is independent of the choice of compatible measurements performed in the measurement context. There has been several attempts to quantify the amount of contextuality for specific quantum systems, for example, in the number of rays needed in a KS proof, or the number of terms in certain inequalities, or in the violation, noise sensitivity, and other measures. This paper is about another approach: to use a simple contextual model that reproduces the quantum-mechanical contextual behaviour, but not necessarily all quantum predictions. The amount of contextuality can then be quantified in terms of additional resources needed as compared with a similar model without contextuality. In this case the contextual model needs to keep track of the context used, so the appropriate measure would be memory. Another way to view this is as a memory requirement to be able to reproduce quantum contextuality in a realist model. The model we will use can be viewed as an extension of Spekkens' toy model [Phys. Rev. A 75, 032110 (2007)], and the relation is studied in some detail. To reproduce the quantum predictions for the Peres-Mermin square, the memory requirement is more than one bit in addition to the memory used for the individual outcomes in the corresponding noncontextual model.
\end{abstract}

Keywords: Contextuality, Quantum Mechanics, Hidden Variables, Finite State Machine PACS: $03.65 . \mathrm{Ta}$

\section{INTRODUCTION}

In this short paper we will see an example of how a noncontextual model, that attempts to emulate quantum mechanics, is modified into a contextual model. The larger and more fundamental question [1]: "Can the quantum-mechanical description be considered complete?" concerns the possibility to augment quantum mechanics (QM) with additional hidden variables (HVs) that provide a more detailed, more complete description (see also [2, 3]). Attempts to construct such HV models include ones where, for a given experiment, the observed probability distributions are used as a HV model [4]. Moreover, there are explicit HV theories, such as Bohmian mechanics [5, 6], which can reproduce all experiments up to date. However, these models use properties that make them less general (using a fundamentally different model for each experimental setup) or undesirable in other ways (superluminal influence). Therefore, we need to make additional assumptions about the structure of the HV model, to avoid this. The most famous result in this direction is Bell's theorem [7], stating that local HV models cannot reproduce the QM correlations between local measurements on some entangled states. That particular set of additional assumptions will not be studied further in this paper but we note that this conflict can be tested in experiment [8-10].

A second seminal result on HV models reproducing QM predictions is the KochenSpecker (KS) theorem [11-13]. This result concerns so-called noncontextual HV mod-

Foundations of Probability and Physics - 6

AIP Conf. Proc. 1424, 211-220 (2012); doi: 10.1063/1.3688973

(C) 2012 American Institute of Physics 978-0-7354-1004-6/\$30.00 
els, in which measurement outcomes do not depend on which other compatible measurements are (have been) performed. There have been several proposals to test the KS theorem in experiment [14-22], but there is also a discussion whether the KS theorem can be experimentally tested at all [23-34]. We will leave that discussion here, and concentrate on one specific noncontextual model, Spekkens' toy model [35], which is known to possess certain QM features but lack others, in particular contextuality. This paper is concerned with an attempt to add contextuality to this model, to make it closer to QM, and analyses the cost of this addition. We will see that the price is to be paid in the form of a larger ontic state space and a more complicated set of rules that govern state changes caused by measurement. The paper starts with a brief description of the system used and the contextuality property of this QM system, and proceeds with a description of Spekkens' toy model and the addition needed to make it contextual.

\section{A QUANTUM SYSTEM AND ITS CONTEXTUALITY}

In this paper, we are going to use a two-qubit system, and measurements on it. When studying questions about local hidden variable models, it is common to assume that the system is in a specific (entangled) state, say the singlet state. Here, we are not going to assume that the system is in such a state, in fact, what follows will be valid even if the state is completely mixed.

We will be concerned with measurement outcomes, and what can be expected to influence these measurement outcomes. Each subsystem can be measured upon by checking whether the "spin of the particle" is up or down in one of the axis directions. In quantum-mechanical terms, our four possible measurement choices (of observables) for each subsystem is $\mathbb{I}, \sigma_{x}, \sigma_{y}$, or $\sigma_{z}$, and these do not commute; for each subsystem we must make one of the four choices. We denote the outcomes +1 for "up" and -1 for "down". We note that there is a difference between doing a separate measurement of $\sigma_{x}$ on the two subsystems, and doing a joint measurement of the product of $\sigma_{x}$ on both. The first corresponds to using the operators $\sigma_{x} \otimes \mathbb{I}$, and $\mathbb{I} \otimes \sigma_{x}$, while the second corresponds to the single operator $\sigma_{x} \otimes \sigma_{x}$, which only outputs one bit of information. We usually assume that this is the sign of the product of the previous outcomes, but one needs to be aware that even this innocuous statement is one of noncontextuality: we assume that the "spin of the first particle along the x-axis" is the same whether we jointly measure the "spin of the second particle along the x-axis" or not.

This is really based in our classical intuition, and the same (noncontextual) reasoning implies that the outcome for one subsystem is independent of measurement choice for the second subsystem. Using classical intuition we expect that the result of measurement of $\sigma_{x} \otimes \mathbb{I}$, should be independent of the choice between $\mathbb{I} \otimes \sigma_{x}, \mathbb{I} \otimes \sigma_{y}$, or $\mathbb{I} \otimes \sigma_{z}$, as a measurement on the second subsystem. This classical intuition underlies the notion

\footnotetext{
1 Classical intuition translates the systems' particle-like behaviour into the belief that we are using particles as our systems. This is not true here, but the present discussion will side-step this important issue. Already spin-1/2 properties are difficult enough, so we will not discuss the more complex issues of position and momentum as properties.
} 
of noncontextuality: The outcome of the measurement of one observable should not be influenced by which simultaneous measurement is performed, which commuting observable that is measured. The two outcomes may be dependent, but the outcome of the first measurement not be influenced by the choice of second measurement.

Perhaps it is in place to stress the connection to counterfactual reasoning: we are saying that "I have measured $\sigma_{x} \otimes \mathbb{I}$ and $\mathbb{I} \otimes \sigma_{x}$, but a noncontextual model would have given the same outcome for the first in the pair if I instead would have measured $\sigma_{x} \otimes \mathbb{I}$ and $\mathbb{I} \otimes \sigma_{z}$ ". A classical system could be expected to obey this, but as we shall see, a quantum system does not.

In this system, one can form the following square of nine observables, also known as the Peres-Mermin (PM) square [36, 37]

$$
\left[\begin{array}{ccc}
\sigma_{z} \otimes \mathbb{I} & \mathbb{I} \otimes \sigma_{z} & \sigma_{z} \otimes \sigma_{z} \\
\mathbb{I} \otimes \sigma_{x} & \sigma_{x} \otimes \mathbb{I} & \sigma_{x} \otimes \sigma_{x} \\
\sigma_{z} \otimes \sigma_{x} & \sigma_{x} \otimes \sigma_{z} & \sigma_{y} \otimes \sigma_{y}
\end{array}\right] .
$$

The square is constructed such that the observables within each row and column commute, corresponding to compatible measurement, and the product of the operators in any row or column yields $\mathbb{I}$, except for the last column, where

$$
\left(\sigma_{z} \otimes \sigma_{z}\right)\left(\sigma_{x} \otimes \sigma_{x}\right)\left(\sigma_{y} \otimes \sigma_{y}\right)=-\mathbb{I} .
$$

In a noncontextual model, the measurement outcomes for each individual measurement would not depend on the choice of compatible measurement from the same row or column. A noncontextual model of our system (containing our two subsystems) would therefore assign \pm 1 values to the outcomes within the matrix, and the products of these values should follow the same rules: the products of the values in any row or column should be +1 , except for the last column where the product should be -1 . But this is impossible. Under the QM rule, multiplying the six row and column products would give the value -1 , but in our noncontextual model, each individual value appears exactly twice (once in each row and once in each column) so that the total product must equal +1 (being a product of squares). Therefore, a noncontextual model must have an even number of $-1 \mathrm{~s}$ in the row and column products, while QM predicts an odd number of -1 s. A noncontextual model cannot obey the QM predictions.

\section{SPEKKENS' TOY MODEL}

We will now briefly shift our focus to one explicit noncontextual model that is specifically designed to mimic QM. It has some of the properties of QM but lacks others. We will just look at the basic construction here, for those interested I would recommend reading [35] and references therein. The toy theory is built on the following foundational principle (The knowledge balance principle):

If one has maximal knowledge, then for every system, at every time, the amount of knowledge one possesses about the ontic state of the system at that time must equal the amount of knowledge one lacks [35]. 
The model is constructed to mimic QM, so in its simplest form, we want to construct the equivalent of a qubit. A qubit measurement has two outcomes, which by the knowledge balance principle means that the ontic state space of a toy bit has four states. The ontic state space can be graphically represented as

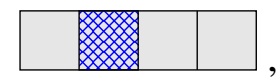

where the ontic state is the second of the four possible. In general, a state can be a statistical mix of these ontic states.

Our measurement can, at the most, identify two out of these four ontic states and say: "the system is in one of these two states" (as opposed to being in one of the other two states). Statements like this identify the epistemic states (the experimentally available outcomes) of the system. There are six combinations of two ontic states, six epistemic states. These naturally form three pairs that can be identified with the three spin-measurement axes
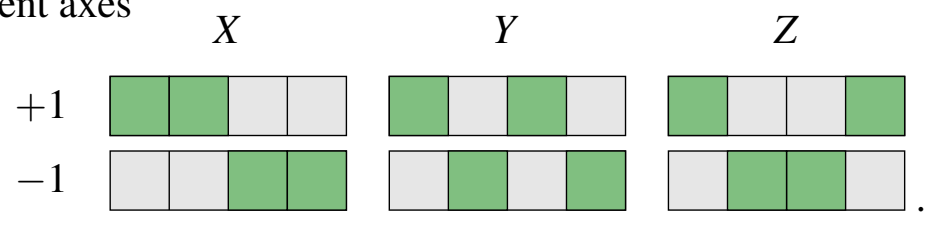

For a system in the ontic state depicted above, a measurement of "the spin along the x-axis" would give the outcome +1 , because the ontic state is contained in the corresponding epistemic state. A subsequent measurement of "the spin along the yaxis" would identify the ontic state completely unless the measurement procedure is allowed to influence the state. Therefore, the knowledge balance principle forces the model to include a state change after measurement. The model is such that the ontic state is randomised after measurement between the two possibilities in the epistemic state. This removes the possibility to extract more information about the ontic state than the knowledge balance principle allows. In this manner, the knowledge balance principle mimics the uncertainty principle from QM.

Having presented the single system construction, we will now quickly review the combination of two such systems into a two-toy-bit model. The ontic state space now has sixteen points, and a specific ontic state can be graphically represented as

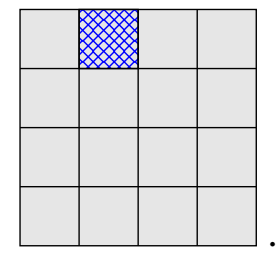

We can maximally extract two bits of knowledge from this system, and the knowledge balance principle gives us many epistemic states, for example

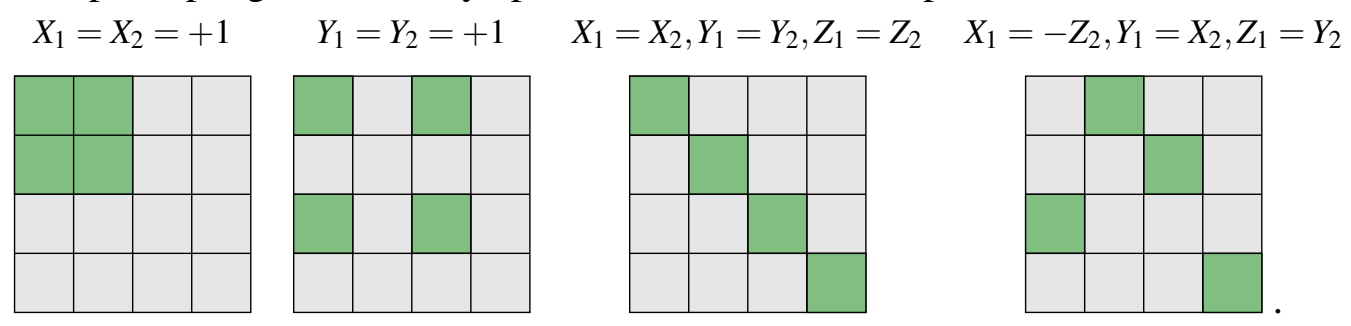


A measurement determining which epistemic state the system is in again randomises between the four ontic states contained in the epistemic state. Note that not all combinations of four ontic states are allowed as epistemic states, again for details, see [35]. This is sufficiently close to two-qubit systems in QM to give several behaviours that can be used to mimic quantum-like behaviour. A partial list would include Noncommutativity (uncertainty), Interference, Remote steering, No cloning, No broadcasting, Mutually Unbiased Partitions, Dense coding, Entanglement monogamy, Teleportation, Positive Operator Valued Measures, .... .

A subsystem measurement on a two-toy-bit model would extract less than maximal information, and the corresponding epistemic states contain eight ontic states. The ontic state of the measured subsystem is randomised, while the ontic state of the unmeasured subsystem is not. In fact, whenever nonmaximal information is extracted, the ontic state is not maximally randomised within the epistemic state, but it is easiest to understand in the example of a subsystem measurement, as in

$$
Z_{1}=+1
$$
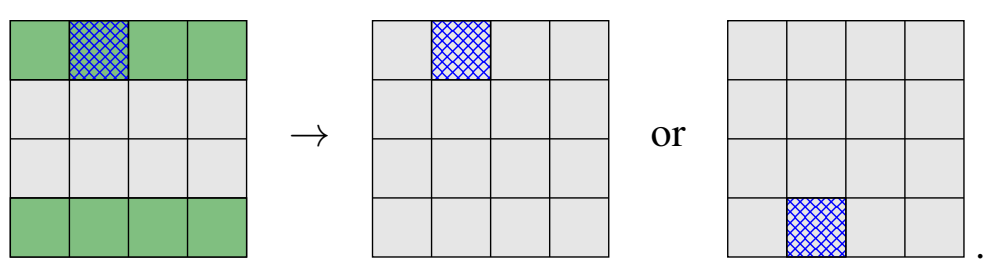

An example of a protocol that uses a two-qu/toy-bit system and performs transformations on one subsystem by itself is dense coding; we can observe that the ontic state space of a one-toy-bit subsystem contains exactly four states, which is just enough to allow for dense coding.

However, not all QM behaviour is captured. The most important missing features are Contextuality, Nonlocality, Continuum of states, Two levels of a three-level system is not a two-level system, "Quantum" computational speedup, ...; we will concentrate on the first item. It is simple to see that the model is noncontextual, because the measurement procedure is such that an outcome of a joint measurement outcome is given by the product of the individual outcomes. Our example ontic state would give the following results:
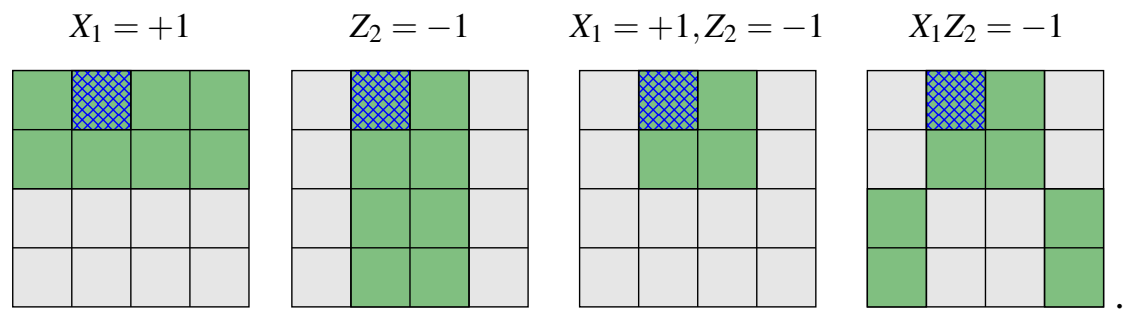

The measurement outcome $X_{1} Z_{2}=-1$ is one of the outcomes of the PM square, when used on the toy model. For this particular ontic state, writing out all the combinations results in

$$
\left[\begin{array}{ccc}
Z_{1} & Z_{2} & Z_{1} Z_{2} \\
X_{2} & X_{1} & X_{1} X_{2} \\
Z_{1} X_{2} & X_{1} Z_{2} & Y_{1} Y_{2}
\end{array}\right]=\left[\begin{array}{ccc}
+1 & -1 & -1 \\
+1 & +1 & +1 \\
+1 & -1 & -1
\end{array}\right] .
$$


Writing out the signs contained in the PM square for each of the ontic states results in

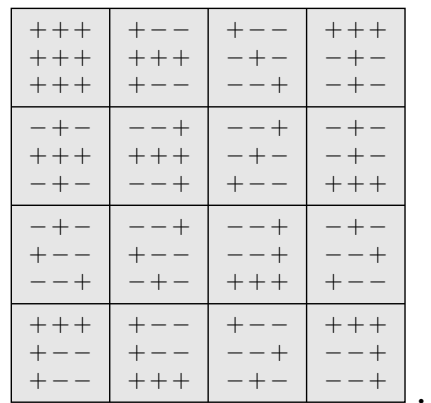

Here, all rows and columns have the product +1 , so that the ontic states can be indexed by the top left four signs of the PM-square values. Also, the last column does not fulfil the $\mathrm{QM}$ prediction, a product equal to -1 .

This can be viewed as a "finite state machine" (specifically, a "stochastic Mealy machine") as follows: each ontic state is one of the sixteen possible states of the machine. When a measurement is performed, the machine outputs a \pm 1 value that depends on its ontic state and the chosen measurement (the "input" to the machine), and then changes state. In the toy model this state change is to a random ontic state within the epistemic state. Below you can see an example, where the large discs are two (ontic) states of the finite state machine and the arrows are state transitions. We start in the left ontic state, perform a measurement of $Z_{1}$ which outputs +1 and changes state randomly to either the same state or a new state with opposite values assigned to the non-commuting observables. The contradiction to the QM prediction is indicated with a dotted ellipse,

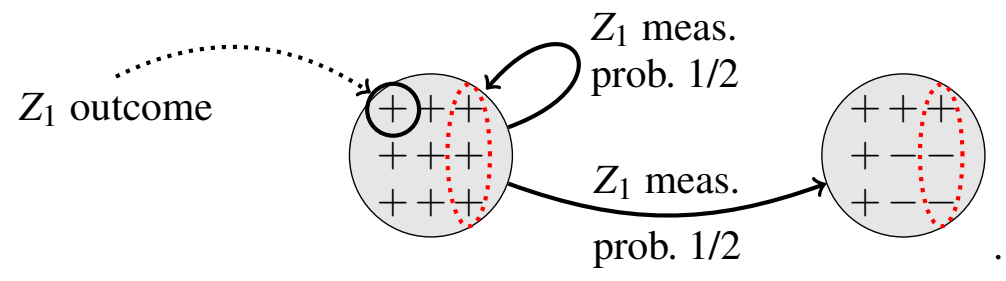

This description is mathematically equivalent to the previous. Of course, the above diagram is only a small part of the state space and transitions, since there are sixteen different ontic states and nine possible measurement choices, all corresponding to a different network of state transitions. Using the language of finite state machines, we can now add properties to the model that were previously difficult to conceptualise. One less desirable effect is that the epistemic states are more difficult to present within the description. But, since our aim is to add properties, we will use the state machine formalism below.

\section{ADDING CONTEXTUALITY TO THE TOY MODEL}

We now want to extend the model so that it gives the QM predictions, including the PM square results. For brevity, we will restrict ourselves to sequential measurements [38], and a more complete presentation of the procedure can be found in [39]. To extend the model properly, we will need to add (ontic) states to the finite state machine. The basic 
questions are what states we add, how many, and if there is a minimal extension. The leftmost state below is already present, and the two others are example states that are possible to add:
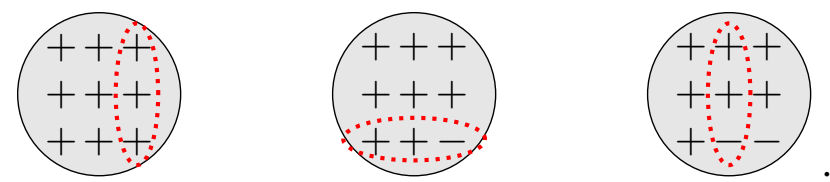

Our starting point will be Spekkens' model, where state transitions always keep the values assigned to commuting observables constant, and randomise the values assigned to non-commuting observables. Our new larger model cannot obey this strictly (although it will preserve the value assigned to the measured observable). This is because of our desire to reproduce the QM predictions for the last column. To do this, some transitions induced by measurement in the last column must change one of the values in the column. Indeed, this transition must go to a state for which the rightmost column product is -1 , as in the below example (we will suppress the randomisation of values of non-commuting observables in what follows)

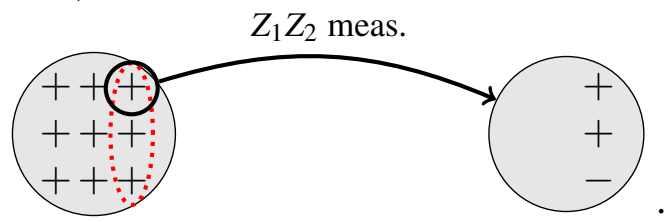

Thus, we will need to add ontic states of the type indicated above. However, it turns out that one transition of this type from the rightmost column is not enough. If, in the above example, we were to perform measurements of the right column observables in the order from the bottom one to the top one, the state change is too late to stop us from finding the column product +1 . Therefore, at least two measurements in the column must give rise to state transitions, both leading to a state with no contradiction in the last column (but a contradiction elsewhere)

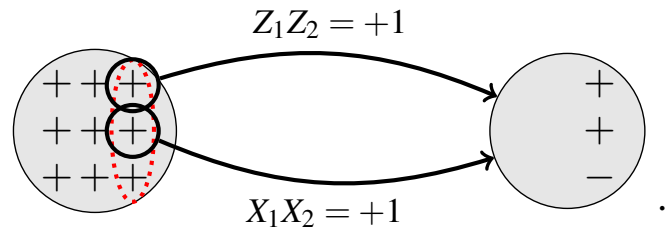

Furthermore, the two transitions must go to different states, because otherwise we will find the column product +1 since the state transition must preserve the value assigned to the measured observable (again, this can be demonstrated by measuring in the order from bottom to top above).

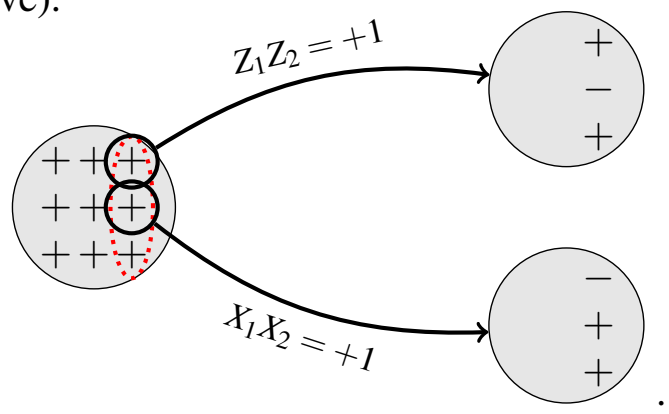


There are now a few alternatives on how to continue to fill in values in the new states. Note that each ontic state still needs to have an even number of -1 row and column products (an assignment of outcomes in a single ontic state must be noncontextual). Therefore, we also need to decide where the -1 product should go among the five alternatives. Some alternatives can be ruled out by using appropriate sequences of measurement choices, but the somewhat tedious procedure [39] will not be repeated here. As it turns out, there is a model with four states that reproduces the QM predictions for the PM square (in the sense that the products are the expected ones)

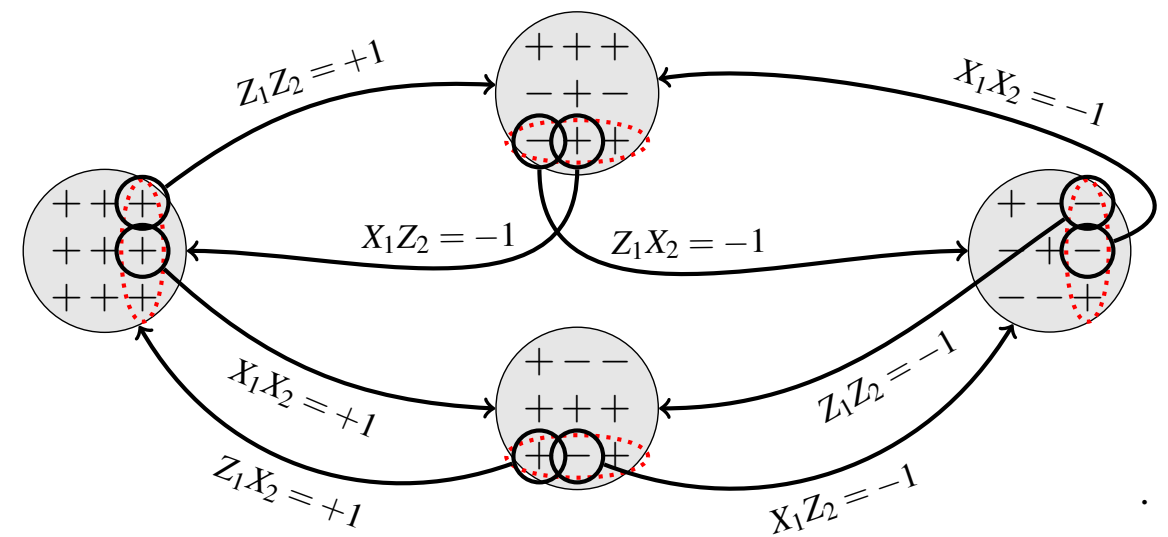

We have added three ontic states to the one we started with, so it is tempting to conclude that the model would need 64 ontic states instead of 16. But a close inspection reveals that the rightmost state above already is present in Spekkens' original model. Further, the two middle states both have their contradiction in the last row, and form a similar class of ontic states. Thus, we only need to add 16 states to Spekkens' model. Our new ontic state space can be depicted by repeating the original table beside another where the bottom right sign has been inverted. The above four-state machine has also been delineated below.

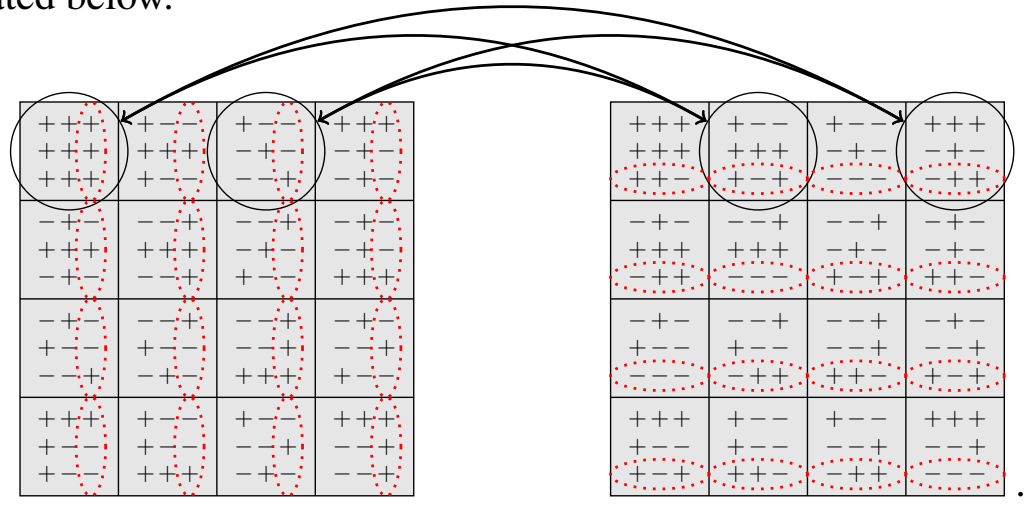

It is a simple matter to include the randomisation of the non-compatible measurement outcomes, but we will skip the details in this short note.

\section{CONCLUSIONS}

Quantum Contextuality is one of the more puzzling properties of QM, so any tool that helps us understand contextuality, ultimately helps us to understand QM. Spekkens' 
toy model is helpful in many ways because it provides a middle ground where we can describe and discuss many of the phenomena associated with QM and Quantum Information Theory, without going to the full-blown QM formalism.

This paper attempts to extend this middle ground to include quantum contextuality into the description and discussion. Using the language of finite state machines, we have successfully included quantum-contextual behaviour. The present note is limited to the case of sequential measurements on pairs of "toy" bits (resembling qubits), and restricts the measurement choices to (the equivalent of) "spin measurements" along two axes. Unfortunately, the lacking third axis means that the model is incomplete, and it seems that the state space needs to be extended even further to incorporate this [39]. Adding a third measurement also adds many relations between the available measurement choices, and results in ten different PM squares [40], instead of one.

Nevertheless, we have seen how to extend Spekkens' toy model to include two more items in the list of quantum-like behaviours that it mimics: Contextuality, and Nonlocality. The latter is included because a two-toy-bit system with separated subsystems would need nonlocal influence to show the needed behaviour: both measurement choices are needed to determine the state transition in the model. One property that is certainly still missing is a continuum of states, but we have yet to see whether two levels of a threelevel system is a two level system or not. We we have not yet seen the three-level version of this new model, so we have nothing to compare with. The possibility of a "Quantum" computational speedup is also still open.

There is a price to pay for this extension, however: the knowledge balance principle fails. There are more than twice as many ontic states as there are epistemic states. Measured in (ordinary) bits of information, the model uses one additional bit of information that keeps track of where the contradiction is in the PM square (last row or last column), and this bit is not accessible to the experimentalist. One way of interpreting this is that the system keeps an internal memory of what the experimenter previously has done to the system. This one bit of information can be proved to be a lower bound for models that obey the QM predictions [39]. Thus, a contextual HV model that gives the QM predictions must obey a knowledge imbalance principle:

If one has maximal knowledge, then for every system, at every time, the amount of knowledge one possesses about the ontic state of the system at that time cannot exceed the amount of knowledge one lacks.

It is the author's firm belief that quantum contextuality is the main cause of the difficulty of understanding QM, and that it plays an important role in Quantum Information Theory. This note (and [39]) provides a link between information theoretical concepts on the one side and quantum contextuality and the Kochen-Specker theorem on the other. There is a definite need to explore the connection further.

\section{ACKNOWLEDGMENTS}

The author would like to thank Adan Cabello, Matthias Kleinmann, Otfried Gühne, Jose Portillo, and Chris Fuchs for a number of interesting discussions. 


\section{REFERENCES}

1. A. Einstein, B. Podolsky, and N. Rosen, Physical Review 47, 777-780 (1935).

2. J. von Neumann, Ann. of Math. 32, 191 (1931).

3. N. Bohr, Physical Review 48, 696-702 (1935).

4. R. F. Werner, and M. M. Wolf, Quantum Information and Computation 1, 1-25 (2001).

5. D. Bohm, and B. J. Hiley, The Undivided Universe, Routledge, London, 1993.

6. P. R. Holland, The Quantum Theory of Motion, Cambridge University Press, Cambridge, UK, 1993.

7. J. S. Bell, Physics (Long Island City, NY) 1, 195 (1964).

8. A. Aspect, J. Dalibard, and G. Roger, Phys. Rev. Lett. 49, 1804 (1982).

9. G. Weihs, T. Jennewein, C. Simon, H. Weinfurter, and A. Zeilinger, Phys. Rev. Lett. 81, 5039 (1998).

10. M. A. Rowe, D. Kielpinski, V. Meyer, W. M. I. C. A. Sackett, C. Monroe, and D. J. Wineland, Nature (London) 409, 791 (2001).

11. E. Specker, Dialectica 14, 239 (1960), english version in The Logico-Algebraic Approach to Quantum Mechanics. Volume I: Historical Evolution, edited by C. A. Hooker (Reidel, Dordrecht, Holland, 1975), p. 135.

12. J. S. Bell, Rev. Mod. Phys. 38, 447 (1966).

13. S. Kochen, and E. P. Specker, J. Math. Mech. 17, 59 (1967).

14. S. M. Roy, and V. Singh, Phys. Rev. A 48, 3379 (1993).

15. A. Cabello, and G. García-Alcaine, Phys. Rev. Lett. 80, 1797 (1998).

16. C. Simon, M. Żukowski, H. Weinfurter, and A. Zeilinger, Phys. Rev. Lett. 85, 1783 (2000).

17. C. Simon, Č. Brukner, and A. Zeilinger, Phys. Rev. Lett. 86, 4427 (2001).

18. J.-Å. Larsson, Europhys. Lett. 58, 799 (2002).

19. A. Cabello, S. Filipp, H. Rauch, and Y. Hasegawa, Phys. Rev. Lett. 100, 130404 (2008).

20. A. A. Klyachko, M. A. Can, S. Binicioğlu, and A. S. Shumovsky, Phys. Rev. Lett. 101, 020403 (2008).

21. A. Cabello, Phys. Rev. Lett. 101, 210401 (2008).

22. P. Badzia̧g, I. Bengtsson, A. Cabello, and I. Pitowsky, Phys. Rev. Lett. 103, 050401 (2009).

23. D. A. Meyer, Phys. Rev. Lett. 83, 3751 (1999).

24. A. Kent, Phys. Rev. Lett. 83, 3755 (1999).

25. N. D. Mermin, A Kochen-Specker theorem for imprecisely specified measurements (1999), quant$\mathrm{ph} / 9912081$

26. R. Clifton, and A. Kent, Proc. R. Soc. London, Ser. A 456, 2101 (2000).

27. H. Havlicek, G. Krenn, J. Summhammer, and K. Svozil, J. Phys. A 34, 3071 (2001).

28. D. M. Appleby, Phys. Rev. A 65, 022105 (2002).

29. A. Cabello, Phys. Rev. A 65, 052101 (2002).

30. T. Breuer, "A Kochen-Specker theorem for unsharp spin 1 observables," in Non-locality and Modality, edited by T. Placek, and J. Butterfield, Kluwer Academic, Dordrecht, Holland, 2002, p. 195.

31. T. Breuer, Phys. Rev. Lett. 88, 240402 (2002).

32. J. Barrett, and A. Kent, Stud. Hist. Phil. Sci. Part B: Stud. Hist. Philos. Mod. Phys. 35, 151 (2004).

33. B. R. La Cour, Phys. Rev. A 79, 012102 (2009).

34. A. Cabello, and J.-A. Larsson, Physics Letters A 375, 99 (2010).

35. R. Spekkens, Phys. Rev. A 75, 032110 (2007).

36. N. D. Mermin, Phys. Rev. Lett. 65, 3373 (1990).

37. A. Peres, Phys. Lett. A 151, 107-108 (1990).

38. J.-̊. Larsson, M. Kleinmann, O. Gühne, and A. Cabello, "Violating noncontextual realism through sequential measurements," in Advances In Quantum Theory: Proceedings Of The International Conference On Advances In Quantum Theory, 2011, vol. 1327 of AIP Conference Proceedings, pp. 401-409.

39. M. Kleinmann, O. Gühne, J. R. Portillo, J.-Å. Larsson, and A. Cabello, New Journal of Physics 13, $113011(2011)$.

40. A. Cabello, Phys. Rev. A 82, 032110 (2010). 